\title{
Nonlinear Compensation Assessment in Few-mode Fibers via Phase-Conjugated Twin Waves
}

\author{
J. S. Tavares, L. M. Pessoa and H. M. Salgado
}

\begin{abstract}
In this paper we further explore the concept of phase-conjugated twin waves (PCTW) for nonlinear cancellation in space-division multiplexed (SDM) systems. Previously, we demonstrated that the PCTW technique can successfully provide nonlinear cancellation in SDM systems. In this paper, we investigate the cases where two and four spatial modes are copropagating in a multimode fiber, considering three link lengths $(1000 \mathrm{~km}, 3200 \mathrm{~km}$ and $8000 \mathrm{~km})$. Weak and strong-coupling regimes are also evaluated. Our numerical simulation results show an average performance improvement $>10 \mathrm{~dB}$ after a 1000 km transmission link.
\end{abstract}

Index Terms-Fiber nonlinearity, multimode fiber, phaseconjugated twin waves, space-division multiplexing.

\section{INTRODUCTION}

W ITH the exponential growth of capacity demand verified in the last few decades, the nonlinear Shannon limit is rapidly being approached [1], [2], [3], [4], [5]. To keep up with the capacity requirements, researchers have explored the available physical dimensions in optical fibers, namely time, phase, frequency and polarization. In order to delay the approaching capacity crunch of single-mode fibers there have been extensive studies that rely on multimode or multicore fibers, which allow multiplexing over different spatial modes of the same fiber by employing spacedivision multiplexing (SDM) [5]. However, alike single-mode fiber based modern telecommunication systems, the nonlinear penalties will eventually become the ultimate limiting factor in SDM systems, resulting from crosstalk effects in multimode or multicore fibers, which generate intramodal and intermodal nonlinearities. Fiber-optic communication systems are largely limited in capacity and reach by fiber Kerr nonlinearity, which leads to distortion when the signal power is increased to achieve adequate signal-to-noise ratio. To overcome this limit, there have been proposed several nonlinearity compensation techniques, such as digital back-propagation (DBP) [6] and mid-link optical phase conjugation (ML-PC) [7]. However, ML-PC requires the transmission link to be modified to include a phase conjugator in the middle of the link, which is impractical, whereas DBP based schemes are known to be computationally complex to implement, even when the interchannel nonlinearities are known to the receiver [8]. Recently, it has been demonstrated that the phase-conjugated twin waves

This work was supported by FCT - Fundação para a Ciência e Tecnologia (Portuguese Foundation for Science and Technology) with the $\mathrm{PhD}$ grant $\mathrm{PD} / \mathrm{BD} / 113821 / 2015$.

The authors are with INESC TEC and Faculty of Engineering, University of Porto, Porto, Portugal (e-mail: joana.tavares@fe.up.pt; luis.m.pessoa@inesctec.pt; hsalgado@fe.up.pt).

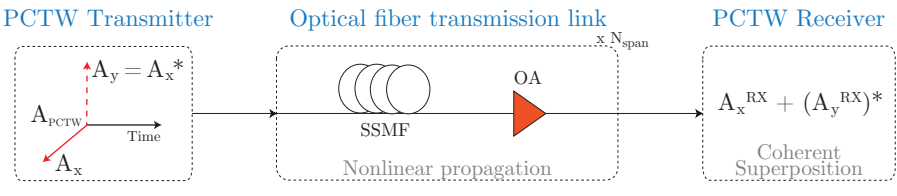

Fig. 1. Single-mode PCTW transmission link diagram.

(PCTW) technique can yield a reduction of nonlinear and linear distortions in single-mode fibers by about $8.5 \mathrm{~dB}$ and $3 \mathrm{~dB}$, respectively [9]. Also, it has been suggested that the concept of PCTW can be further extended by using an orthogonal dimension rather than the orthogonal polarization to transmit the complex conjugate (e.g. space). Additionally, the same can be applied to vector waves, where the signal and its conjugated twin are PDM (polarization-division multiplexed) signals (consisting of two independent polarization components), propagating on orthogonal dimensions (e.g. space) [9], [10].

In a previous work we proposed three multimode PCTW scenarios, that were based on PDM-wise and mode-wise approaches [11]. Our numerical simulations showed that PCTW can successfully be used for nonlinear mitigation in SDM systems as well. Moreover, the PDM-wise approach provided better results, yielding a performance improvement of $\sim 10 \mathrm{~dB}$, when compared with the single-mode PCTW case.

In this work we continue exploring nonlinearity mitigation in SDM systems by employing the PCTW technique. Specifically, we assessed the impact on system performance when copropagating two and four spatial modes, in the weak and strong coupling regimes. Furthermore, the impact of transmission length was also assessed.

The remainder of this paper is organized as follows: in Section II we first describe the concept of PCTW. In Section III we describe the multimode PCTW configurations which were assessed. Section IV comprises the numerical simulation model including simulation parameters. In Section V the obtained numerical simulation results are presented and discussed. Lastly, the final conclusions are given in Section VI.

\section{Single-MOde PCTW}

PCTW-based transmission in single-mode fibers has been previously proposed [9]. This technique consists of propagating a PDM optical signal, where one of the orthogonal polarization components is the complex conjugate of the other, as depicted in Fig. 1. At the receiver side, the nonlinear distortions are cancelled to first order by coherently superimposing the received twin waves. It has been proved that the nonlinear 
TABLE I

MULTIMODE PCTW CONFIGURATIONS.

2 Spatial Modes

distortions experienced by a pair of mutually PCTWs are anti-correlated, which leads to the first-order cancellation of nonlinear interactions upon coherently superimposing the received signals [9]. This technique requires a symmetric dispersion map, which could be readily applied in a dynamic optical network environment, through the employment of electronic dispersion pre-compensation at the transmitter. This technique has the potential to reduce the overall link costs, specially in applications such as ultralong-haul transmission and transoceanic optical links, since it allows a reduction of the number of transceivers by $50 \%$. On the downside, with this technique spectral efficiency is traded for transmission performance [9].

The nonlinear propagation in a single-mode fiber can be described by the well-known Manakov-PMD equations [12], [13], [14], as follows:

$$
\frac{\partial A_{\mathrm{PCTW}}}{\partial z}+i \frac{\beta_{2}}{2} \frac{\partial^{2} A_{\mathrm{PCTW}}}{\partial t^{2}}=i \frac{8}{9} \gamma\left(\left|A_{x}\right|^{2}+\left|A_{y}\right|^{2}\right) A_{\mathrm{PCTW}},
$$

where $\beta_{2}$ is the group velocity dispersion (GVD), $\gamma$ is the Kerr nonlinear parameter. The PDM signal containing the twin wave is given by

$$
A_{\mathrm{PCTW}}=\left(A_{x}, A_{y}\right)^{T},
$$

consisting of

$$
\left\{\begin{array}{l}
A_{x}=A \\
A_{y}=A_{x}^{*}
\end{array}\right.
$$

At the receiver side, the original signal is recovered without nonlinear distortions (to first order) after coherent superposition of the received signals [9], by employing

$$
A_{x}^{R X}+\left(A_{y}^{R X}\right)^{*}=2 A .
$$

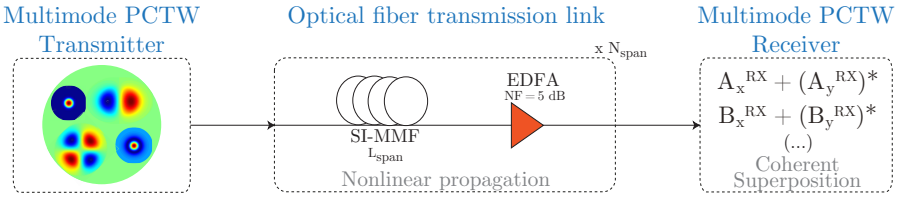

Fig. 2. Multimode PCTW transmission link diagram.

\section{Multimode PCTW}

Table I shows the investigated multimode PCTW configurations, following a PDM-wise approach [11], where each pair of PCTWs is propagated on a single spatial mode and different pairs are sent on distinct spatial modes. Here, we investigate the case considering two spatial modes (i.e. LP01 and LP11) and four spatial modes (i.e. LP01, LP11, LP02 and LP21). At the receiver side the received twin waves are coherently super-positioned accordingly to each spatial mode, as shown in Fig. 2. However, Eq. (1) cannot be used to describe nonlinear propagation in multimode fibers since it does not include the intermodal nonlinear effects. An extension of the standard Manakov equation (SMF based) to multimode fibers was proposed and evaluated in [15], by assuming that the state of polarization of each spatial mode evolves randomly and independently from the other modes. This equation governs propagation of arbitrarily polarized light in the spatial modes, and includes all nonlinear effects (intramodal as in SMF as well as intermodal nonlinearities among various fiber spatial modes), random polarization birefringence, chromatic dispersion and fiber losses within each spatial mode. Moreover, the authors derived a set of equations regarding the weak and strong-coupling regimes. In general, coupling strength between spatial modes can vary for different mode pairs. For instance, the LP11a and LP11b spatial modes are strongly coupled due to its degenerated nature, while the modes LP01 and LP11 are weakly coupled [16]. However, in this work the two coupling regimes represent two extreme cases for practical systems, where in the weak-coupling regime the linear coupling between spatial modes is disregarded, while in the strong coupling regime it is assumed that all modes are strongly coupled.

In the weak-coupling regime [15], the nonlinear propagation for the $p^{\text {th }}$ mode is given by:

$$
\begin{aligned}
& \frac{\partial \bar{A}_{p}}{\partial z}+\left\langle\delta \beta_{0 p}\right\rangle \bar{A}_{p}+\left\langle\delta \beta_{1 p}\right\rangle \frac{\partial \bar{A}_{p}}{\partial t}+i \frac{\beta_{2 p}}{2} \frac{\partial^{2} \bar{A}_{p}}{\partial t^{2}} \\
= & i \gamma\left(f_{p p p p} \frac{8}{9}\left|\bar{A}_{p}\right|^{2}+\sum_{m \neq p} f_{m m p p} \frac{4}{3}\left|\bar{A}_{m}\right|^{2}\right) \bar{A}_{p},
\end{aligned}
$$

where $\gamma$ is the Kerr nonlinear parameter, $\left\langle\delta \beta_{0 p}\right\rangle$ is the propagation constant, $\left\langle\delta \beta_{1 p}\right\rangle$ is the DMGD and $\beta_{2 p}$ is the GVD. The nonlinear coefficient $f_{l m n p}$ provides a relative weight among the nonlinear terms between the spatial modes and is defined by:

$$
f_{l m n p}=\frac{A_{\mathrm{eff}}}{\left(I_{l} I_{m} I_{n} I_{p}\right)^{1 / 2}} \iint F_{l}^{*} F_{m} F_{n} F_{p}^{*} d x d y,
$$

where $I_{l}, I_{m}, I_{n}$ and $I_{p}$ represent the constants of normalization of the modal fields $F_{l}, F_{m}, F_{n}$ and $F_{p}$, where the indices 
TABLE II

INVERSE GROUP VELOCITY, DIFFERENTIAL MODE GROUP DELAY AND DISPERSION FOR THE DIFFERENT MODES.

\begin{tabular}{ccccc}
\hline & $\mathrm{LP}_{01}$ & $\mathrm{LP}_{11}$ & $\mathrm{LP}_{02}$ & $\mathrm{LP}_{21}$ \\
\hline$\beta_{1}(\mathrm{~ns} / \mathrm{km})$ & 4901.7 & 4908.2 & 4911.6 & 4914.1 \\
$\mathrm{DMGD}(\mathrm{ns} / \mathrm{km})$ & 0 & 6.5 & 9.9 & 12.4 \\
$\mathrm{D}(\mathrm{ps} / \mathrm{nm} / \mathrm{km})$ & 25.1 & 27.4 & -2.5 & 20.9 \\
$\mathrm{~A}_{\text {eff }}\left(\mu \mathrm{m}^{2}\right)$ & 80 & 76.4 & 83.5 & 86.5 \\
\hline
\end{tabular}

represent the $\mathrm{M}$ spatial modes. $A_{\mathrm{eff}}$ is the effective area of the fundamental mode, given by Eq. (7).

$$
A_{\mathrm{eff}}=\frac{\left(\iint\left|F_{m}\right|^{2} d x d y\right)^{2}}{\iint\left|F_{m}\right|^{4} d x d y} .
$$

In the strong-coupling regime [15], the nonlinear propagation is described by

$$
\frac{\partial \bar{A}}{\partial z}+i \frac{\bar{\beta}_{2}}{2} \frac{\partial^{2} \bar{A}}{\partial t^{2}}=i \gamma \kappa|\bar{A}|^{2}|\bar{A}|,
$$

where the nonlinear coefficient $\kappa$ is given by Eq. (9).

$$
\kappa=\sum_{k \leq l}^{M} \frac{32}{2^{\delta_{k l}}} \frac{f_{k k l l}}{6 M(2 M+1)} .
$$

Furthermore, this new set of Manakov equations drastically reduces computational time, since nonlinear terms are minimized [15].

\section{Numerical Simulations}

In this section we describe our numerical simulation model.

At the transmitter side, a signal generated by a pseudo random binary sequence (PRBS) was modulated in a QPSK carrier. We considered 32-Gbaud signals (single-channel), consisting of $2^{13}$ modulated symbols. The complex conjugate of this signal was computed to form the PCTW signal. Before transmission, the signals were filtered by a squared root raised cosine (SRRC) filter with a 0.1 roll-off factor and predispersion compensated for half of the link length.

Regarding the transmission medium, we considered a stepindex few-mode fiber (FMF), with a core radius of $6 \mu \mathrm{m}$ and a fiber loss of $0.2 \mathrm{~dB} / \mathrm{km}$. The nonlinear coefficient, numerical aperture and V parameter at $1550 \mathrm{~nm}$ used were 1.4 $\mathrm{W}^{-1} \mathrm{~km}^{-1}, 0.2$, and 5, respectively. Such optical fiber supports the linearly polarized modes LP01, LP11, LP02 and LP21, that is to say it supports up to six spatial modes considering the 2fold degeneracy of LP11 and LP21 [17]. Inverse group velocity $\left(\beta_{1}\right)$, differential mode group delay (DMGD), dispersion (D) and effective area $\left(\mathrm{A}_{\text {eff }}\right)$ calculated for each mode are given in Table II [17], [18], [19], [20], [21]. For comparison, we also considered a SMF with different characteristics from the multimode fiber, specifically having a V-parameter of 2.2, a dispersion of $17 \mathrm{ps} / \mathrm{nm} / \mathrm{km}$ and nonlinear parameter of 1.27 $\mathrm{W}^{-1} \mathrm{~km}^{-1}$. This step index SMF has an effective area of 142 $\mu \mathrm{m}^{2}$, a core radius $6 \mu \mathrm{m}$ and a fiber loss of $0.2 \mathrm{~dB} / \mathrm{km}$.

The numerical simulations were performed for three different transmission lengths: $1000 \mathrm{~km}$ (10x100-km fiber spans), $3200 \mathrm{~km}$ (40x80-km fiber spans) and $8000 \mathrm{~km}(100 x 80-\mathrm{km}$ fiber spans). The losses in each fiber span were completely compensated by an erbium-doped fiber amplifier (EDFA), that also added ASE (amplified spontaneous emission) noise, with a noise figure of $5 \mathrm{~dB}$.

Regarding multimode propagation, weak and strongcoupling regimes were assessed using Eq. (5) and Eq. (8), respectively, while single mode propagation was assessed using the SMF Manakov equation (1). These equations were solved using the standard symmetric split-step Fourier method [22].

In the multimode case, the power was injected into the different modes simultaneously in such a way that they copropagate in the fiber, considering two and four modes, as indicated in Table I. Here, the launch power is the power per mode and thus, compared to the SMF-case, the required total launch power is two times higher in the 2-mode case and four times higher in the 4-mode case.

At the receiver side, the received signals were postdispersion compensated for the rest of the fiber link, thus realising the symmetric dispersion map required to attain the full benefit of the PCTW technique [9], followed by SRRC filtering. Phase and constellation rotation of the received twin waves were calculated and corrected before coherent superposition, using Eq. (4). Finally, the received signals were demodulated.

The metric used to evaluate the performance of the simulated PCTW configurations was the $\mathrm{Q}^{2}$-factor, using the definition proposed in [23]. System performance was determined by calculating the $\mathrm{Q}^{2}$-factor of the received signals, as well as the average $\mathrm{Q}^{2}$-factor in the multimode case, before and after superposition, for each launch power.

\section{Numerical Simulation Results}

System performance of the multimode PCTW was assessed, by propagating two and four pairs of PCTWs, co-propagating on two and four distinct spatial modes, according to Table I, for three different transmission link lengths $(1000 \mathrm{~km}, 3200$ $\mathrm{km}$ and $8000 \mathrm{~km}$ ).

The results obtained in the weak-coupling regime are presented in Fig. 3 and Fig. 4, where two and four pairs of PCTWs were propagated, respectively, showing the performance of the individual modes, as well as the average performance. Our results include the multimode and SMF cases, before (i.e. PDM signals) and after (i.e. PCTW signals) coherent superposition. Here, PDM is the result obtained for a dual polarization signal, where the $y$-polarization contains the complex conjugate of the x-polarization, and PCTW is the result after coherent superposition of the two received PDM signals. Fig. 5 and Fig. 6 show the same results in the strong-coupling regime.

Our numerical results show that, on average, multimode propagation performs better than the single-mode case. Multimode fibers present lower nonlinearity due to higher effective 


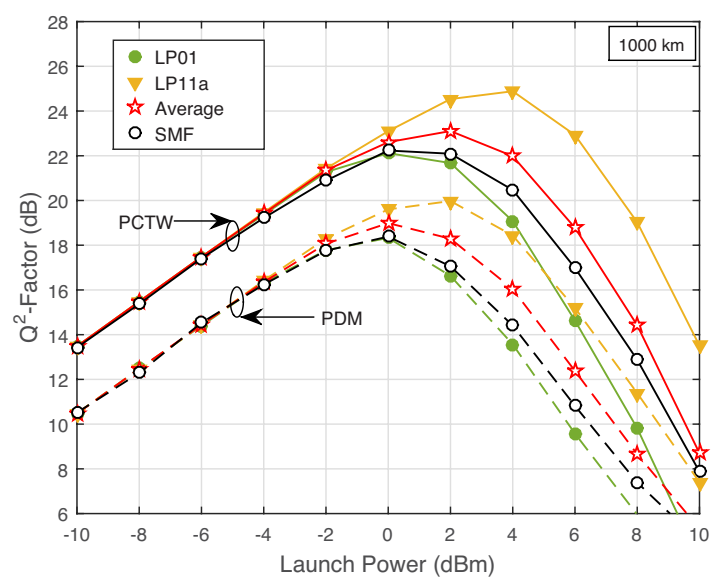

(a)

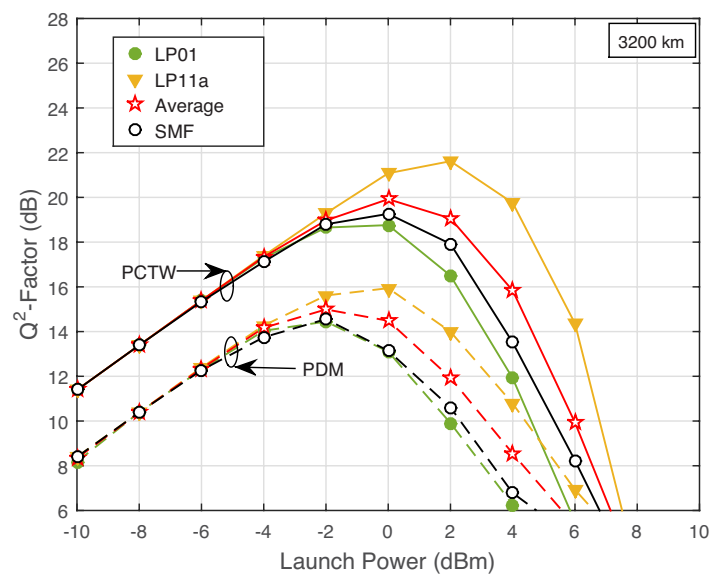

(b)

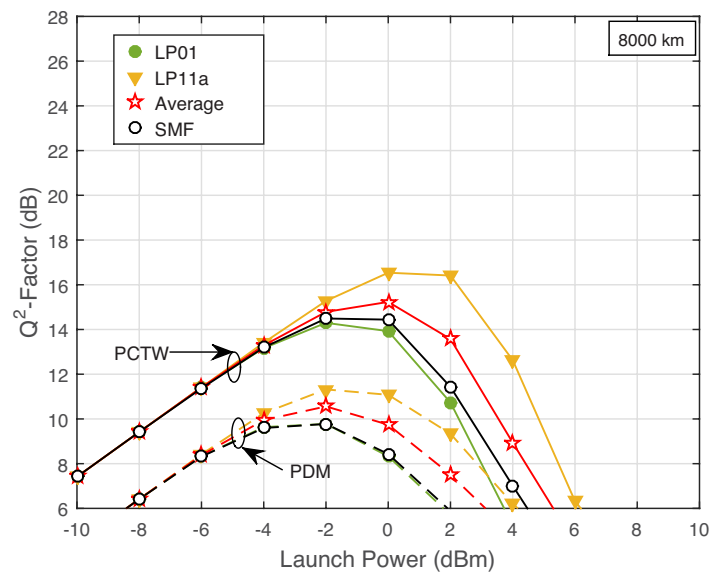

(c)

Fig. 3. Performance of two individual co-propagating modes in the weak coupling regime, before (PDM, dashed line) and after (PCTW, solid line) coherent superposition of the received signals, as well as the average (pentagram) and single mode (blank circle) performance. Transmission length: (a) $1000 \mathrm{~km}$, (b) $3200 \mathrm{~km}$ and (c) $8000 \mathrm{~km}$.

area, therefore these present better performance compared to SMF [24]. Moreover, DMGD reduces the intermodal nonlinear effects, since it induces de-correlation between spatial modes, thus the impact of nonlinear effects is mitigated [25].

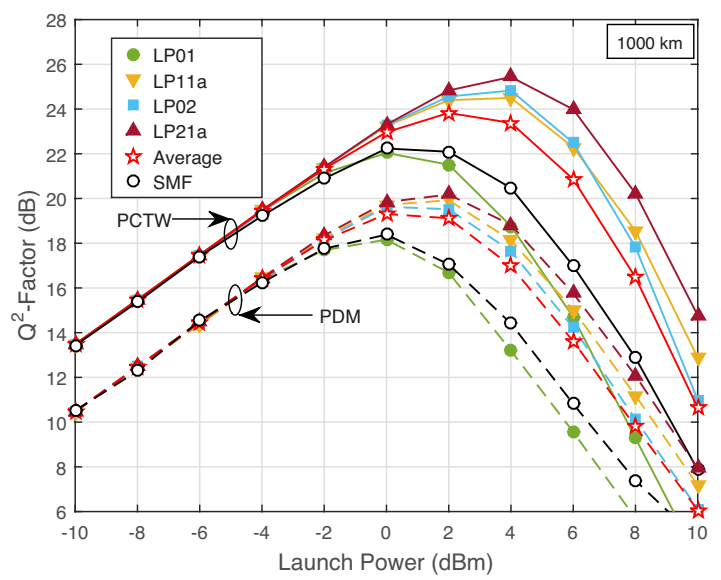

(a)

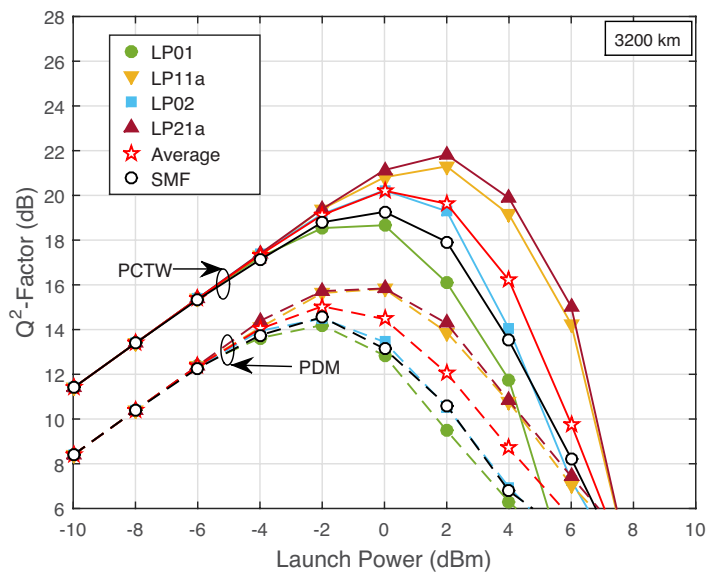

(b)

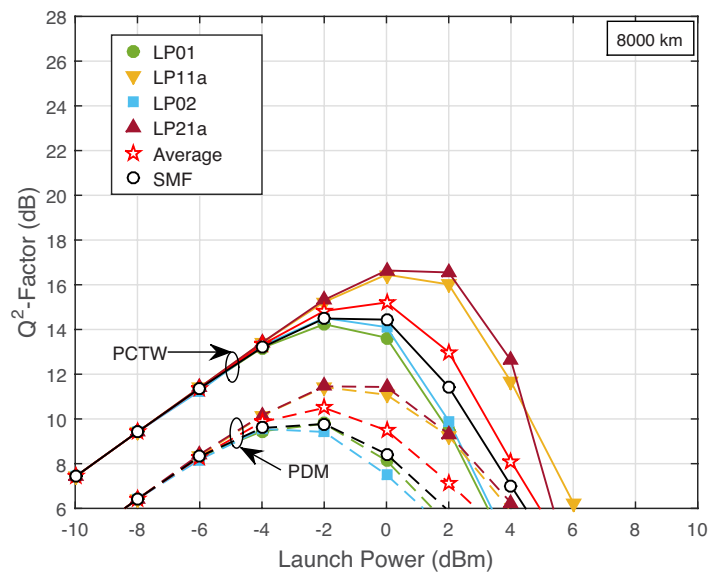

(c)

Fig. 4. Performance of four individual co-propagating modes in the weak coupling regime, before (PDM, dashed line) and after (PCTW, solid line) coherent superposition of the received signals, as well as the average (pentagram) and single mode (blank circle) performance. Transmission length: (a) $1000 \mathrm{~km}$, (b) $3200 \mathrm{~km}$ and (c) $8000 \mathrm{~km}$.

Note that, in the weak-coupling regime (Fig. 3 and Fig. 4), the $\mathrm{Q}^{2}$-factors of individual modes depend on many factors, such as the nonlinear coefficients, the dispersion parameter and the DMGD. Some conclusions regarding the relative 


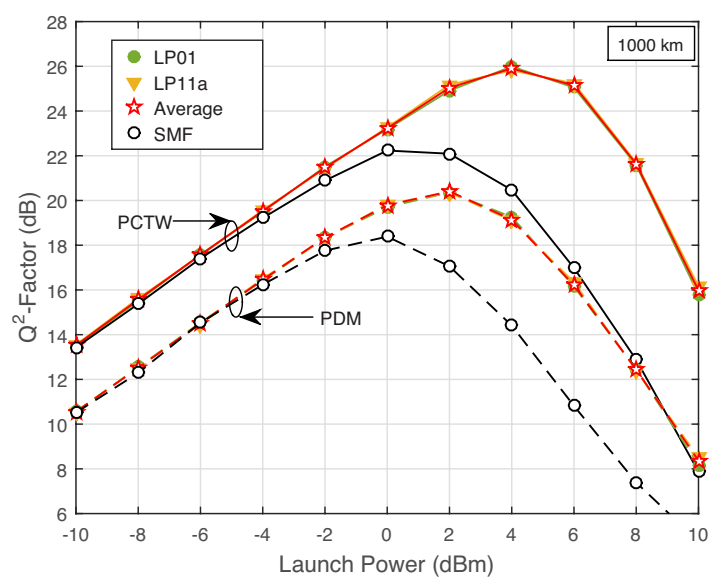

(a)

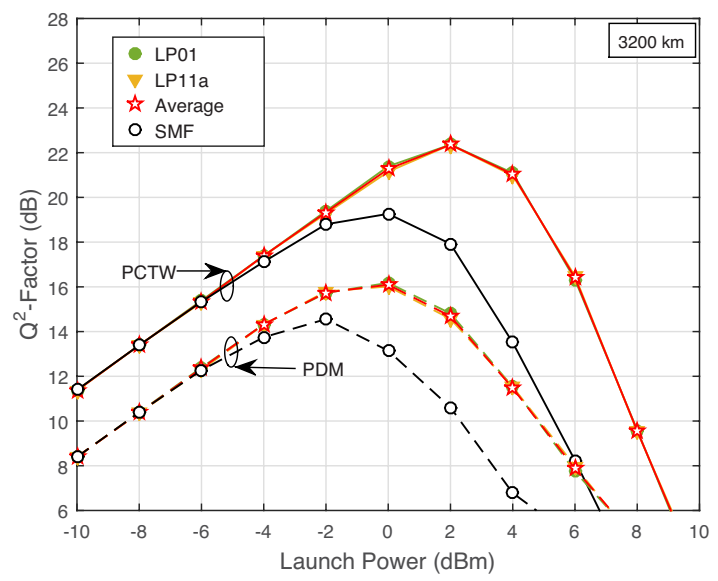

(b)

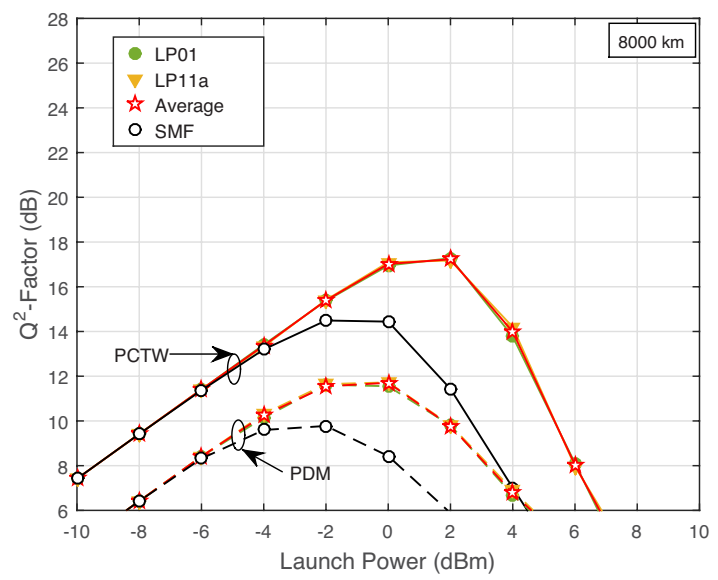

(c)

Fig. 5. Performance of two individual co-propagating modes in the strong coupling regime, before (PDM, dashed line) and after (PCTW, solid line) coherent superposition of the received signals, as well as the average (pentagram) and single mode (blank circle) performance. Transmission length: (a) $1000 \mathrm{~km}$, (b) $3200 \mathrm{~km}$ and (c) $8000 \mathrm{~km}$.

performance of the various modes and a comparison with previous results, where existent, follows. Regarding the two mode propagation (Fig. 3), we verify that LP11 mode performs better than LP01, which agrees with previous works [24].

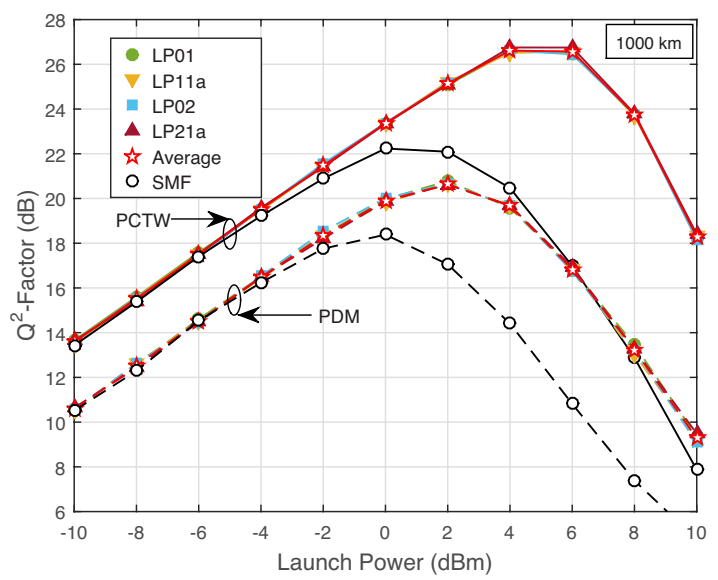

(a)

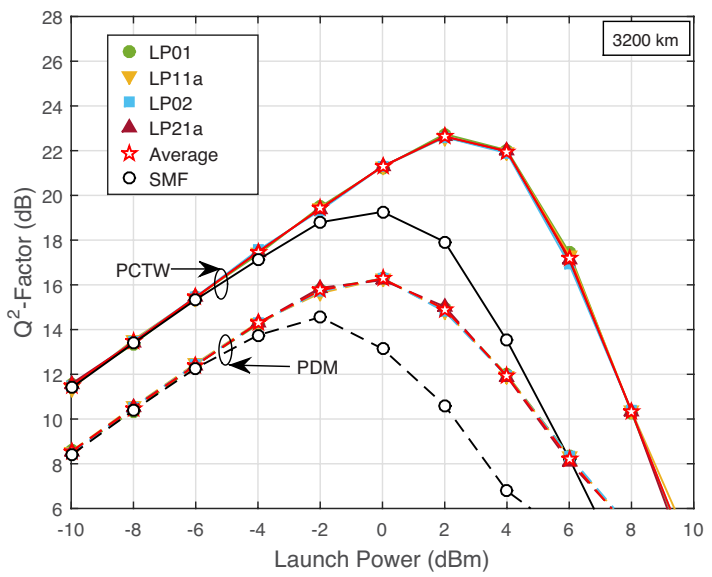

(b)

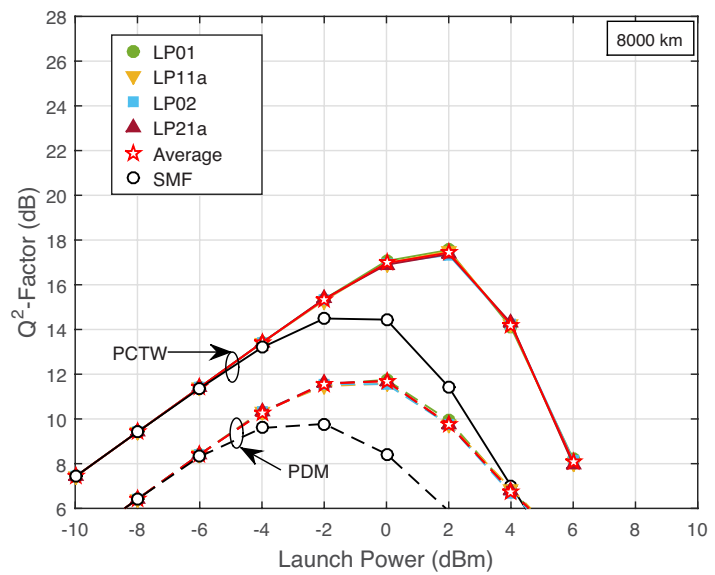

(c)

Fig. 6. Performance of four individual co-propagating modes in the strong coupling regime, before (PDM, dashed line) and after (PCTW, solid line) coherent superposition of the received signals, as well as the average (pentagram) and single mode (blank circle) performance. Transmission length: (a) $1000 \mathrm{~km}$, (b) $3200 \mathrm{~km}$ and (c) $8000 \mathrm{~km}$.

Regarding the four mode propagation (Fig. 4), the results show that the degenerate modes LP11a and LP21a present the best performance. The low dispersion of the LP02 mode leads to a clear degradation of this mode with distance when compared 

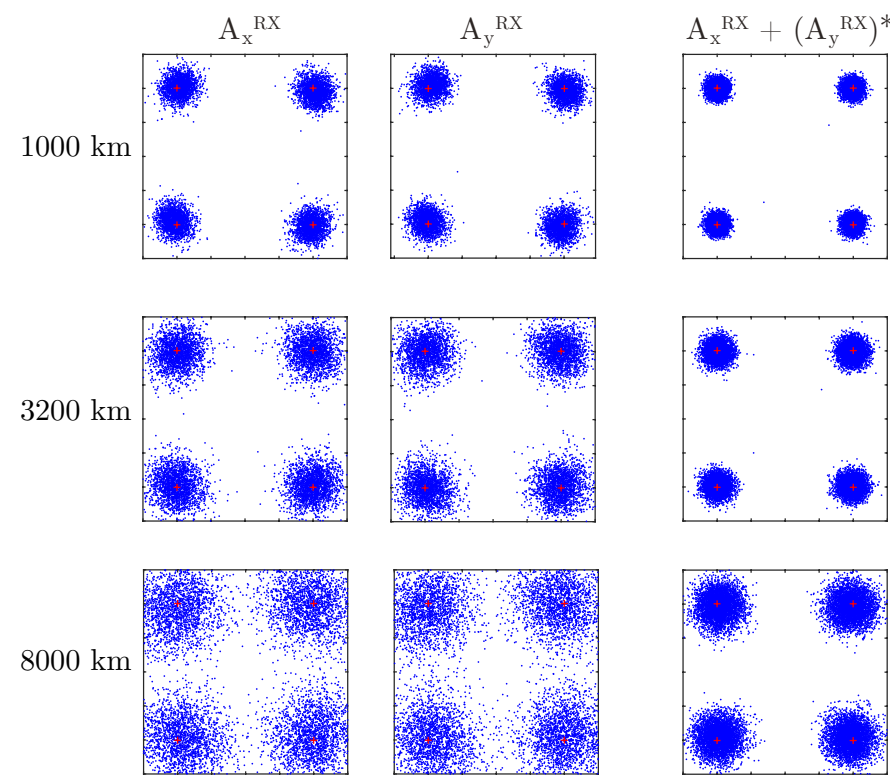

Fig. 7. Recovered constellations before and after coherent superposition of the received LP01 mode, for the different transmission lengths at optimum launch power ( $2 \mathrm{dBm}, 0 \mathrm{dBm}$ and $0 \mathrm{dBm}$, respectively).

to the other modes. Although the pre-dispersion compensation shifts the point of zero accumulated dispersion to the middle of the link, a point where the accumulated noise helps to average out the effect of nonlinearity, this is not enough to mitigate the impact of nonlinearity during the propagation of signals with small dispersion, which is naturally aggravated for longer distances. Moreover, the performance of LP01 in the multimode is somewhat worse than in the SMF, since the SMF that we considered has distinct characteristics as described in Section IV.

In the strong-coupling regime (Fig. 5 and Fig. 6), all spatial modes propagate, on average, with the same group velocity, which results in having the same performance for all the modes. Also, the optimal launch power increased for all cases.

Fig. 7 shows the constellations of the received LP01 mode obtained at the optimum signal power $(2 \mathrm{dBm}, 0 \mathrm{dBm}$ and 0 $\mathrm{dBm})$ for the different transmission lengths $(1000 \mathrm{~km}, 3200$ $\mathrm{km}$ and $8000 \mathrm{~km}$ ), respectively, in the weak-coupling regime, showing the effectiveness of the PCTW technique.

The average $\mathrm{Q}^{2}$-factor improvement achieved by the PCTW technique (i.e. the difference between before and after coherent superposition), for the different link lengths, is presented in Fig. 8 and Fig. 9, for the weak and strong coupling regimes, respectively. These results show that the strong-coupling regime, on average, performs better than the weak-coupling regime, as previously demonstrated [11], [15]. Note that, the weak and strong coupling regimes in this work represent two extreme cases. In practice, the achieved multimode performance will be between these two cases. Multimode fibers, in general, present some level of coupling between any pair of modes, arising from different sources, such as manufacturing defects (variations in the core radius), bending, twisting, and other [26]. In the linear (noise limited) region the performance improvement is around $3 \mathrm{~dB}$ for all cases, as expected. In

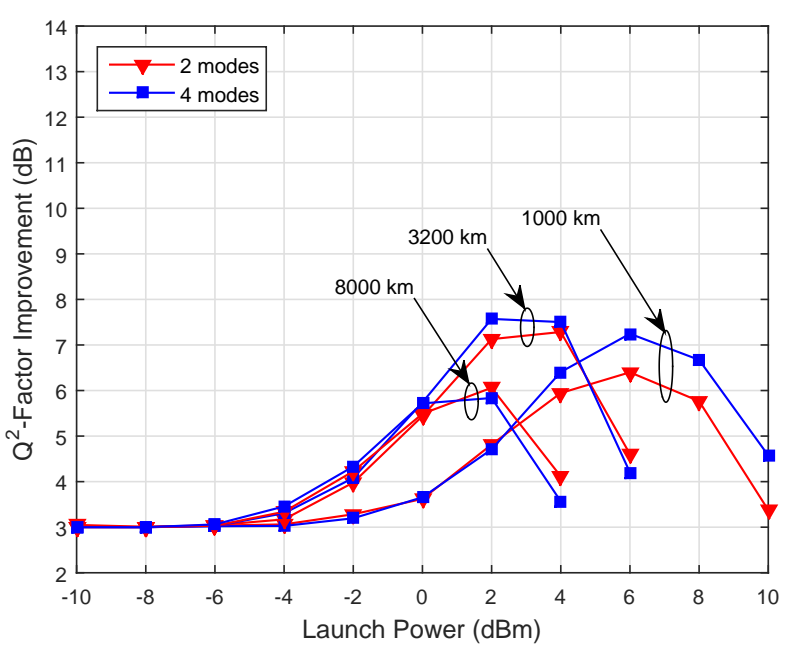

Fig. 8. Average $\mathrm{Q}^{2}$-factor improvement for the different link lengths, in the weak coupling regime.

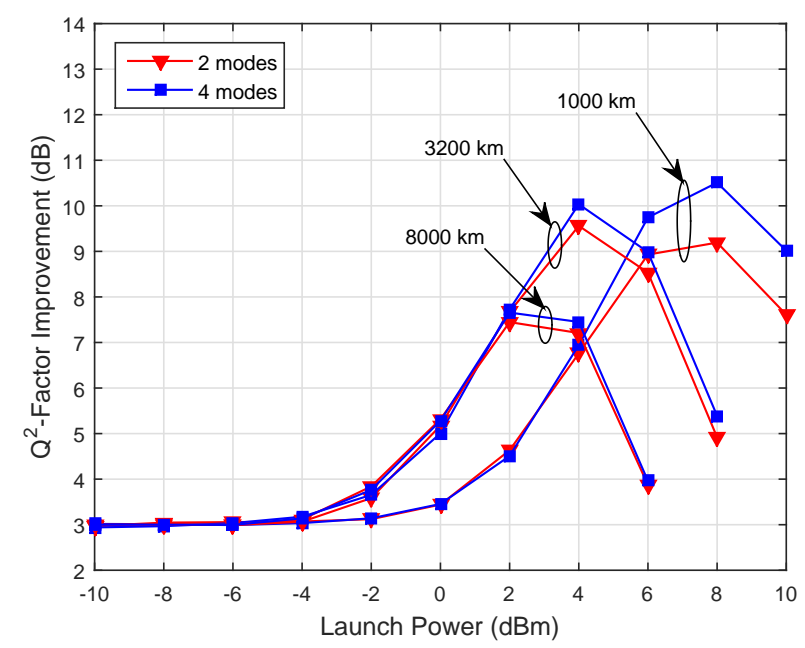

Fig. 9. Average $\mathrm{Q}^{2}$-factor improvement for the different link lengths, in the strong coupling regime.

Fig. 8 we verify that in the weak-coupling regime the average performance improvement achieved with the PCTW technique goes up to 6 and $7 \mathrm{~dB}$ after a $1000 \mathrm{~km}$ transmission link, regarding 2 and 4 spatial modes, respectively. After 3200$\mathrm{km}$ the average $\mathrm{Q}^{2}$-improvement goes up to $\sim 7.5 \mathrm{~dB}$. Here, the improved performance compared to the $1000 \mathrm{~km}$ results is due to a smaller span length (decreased from $100 \mathrm{~km}$ to $80 \mathrm{~km}$ ). Finally, after $8000 \mathrm{~km}$ the average performance improvement is around $6 \mathrm{~dB}$, showing a smaller improvement, since after a much longer transmission the received signal is degraded by noise, thus the algorithm is not so effective. The strong-coupling regime (Fig. 9) shows an average performance improvement between 9 and $10.5 \mathrm{~dB}$ after $1000 \mathrm{~km}$, considering 2 and 4 spatial modes, respectively. After 3200 $\mathrm{km}$, the average performance improvement goes up to 9.5$10 \mathrm{~dB}$. Lastly, after $8000 \mathrm{~km}$ the average $\mathrm{Q}^{2}$-improvement is around $7.5 \mathrm{~dB}$, again showing a smaller improvement after 
a much longer transmission length. On average, four copropagating modes perform better than two modes after a $1000 \mathrm{~km}$ transmission, whereas for longer transmission lengths that difference vanishes, since that for longer distances the received signal is degraded by noise and the algorithm is not so effective.

\section{CONCLUSION}

The performance of SDM-PCTW has been assessed numerically for different co-propagating modes and transmission lengths. Our numerical simulation results show that copropagating four spatial modes provides better performance than two spatial modes after a $1000 \mathrm{~km}$ fiber transmission, whereas for longer distances that difference is much smaller. PCTW further improves SDM performance by $7 \mathrm{~dB}$ in the weak coupling regime and $10 \mathrm{~dB}$ in the strong coupling regime, after a $1000 \mathrm{~km}$ transmission link. Moreover, for much longer transmission links $(8000 \mathrm{~km})$, the benefit of this technique is still significant, showing a Q-improvement around 6-7 dB.

\section{REFERENCES}

[1] R.-J. Essiambre and A. Mecozzi, "Capacity limits in single mode fiber and scaling for spatial multiplexing," in Optical Fiber Communication Conference and Exposition and National Fiber Optic Engineers Conference (OFC/NFOEC), Optical Society of America, 2012, OW3D.1.

[2] P. J. Winzer, "Challenges and evolution of optical transport networks," in European Conference on Optical Communication (ECOC), pp. 19-23, 2010, We.8.D.1.

[3] P. J. Winzer, "Energy-efficient optical transport capacity scaling through spatial multiplexing," Photonics Technology Letters, IEEE, vol. 23, no. 13 , pp. 851-853, 2011.

[4] P. J. Winzer and G. J. Foschini, "MIMO capacities and outage probabilities in spatially multiplexed optical transport systems," Optics Express, vol. 19, no. 17, pp. 16680-16696, 2011.

[5] D. J. Richardson, J. M. Fini, and L. E. Nelson, "Space-division multiplexing in optical fibres," Nature Photonics, vol. 7, no. 5, pp. 354-362, 2013.

[6] L. M. Pessoa, H. M. Salgado, and I. Darwazeh, "Assessment of dispersion map impact on the digital back propagation of long-haul fibre-optic links," Optical and Quantum Electronics, vol. 45, no. 3, pp. 257-269, 2013.

[7] X. Chen, X. Liu, S. Chandrasekhar, B. Zhu, and R. W. Tkach, "Experimental demonstration of fiber nonlinearity mitigation using digital phase conjugation," in Optical Fiber Communication Conference and Exposition and National Fiber Optic Engineers Conference (OFC/NFOEC), pp. 1-3, 2012, OTh3C.1.

[8] D. Rafique and A. Ellis, "Scaling the advantages of intra-channel nonlinearity compensation in future flexible optical networks," in European Conference on Optical Communication (ECOC), p. P4.18, Optical Society of America, 2012.

[9] X. Liu, A. R. Chraplyvy, P. J. Winzer, R. W. Tkach, and S. Chandrasekhar, "Phase-conjugated twin waves for communication beyond the Kerr nonlinearity limit," Nature Photonics, vol. 7, no. 7, pp. 560-568, 2013.

[10] X. Liu, S. Chandrasekhar, P. J. Winzer, R. W. Tkach, and A. R. Chraplyvy, "Fiber-nonlinearity-tolerant superchannel transmission via nonlinear noise squeezing and generalized phase-conjugated twin waves," Journal of Lightwave Technology, vol. 32, no. 4, pp. 766-775, 2014.

[11] J. S. Tavares, L. M. Pessoa, and H. M. Salgado, "Phase conjugated twin waves based transmission in few modes fibers," in International Conference on Transparent Optical Networks (ICTON), pp. 1-4, 2015, We.D1.3.

[12] D. Marcuse, C. R. Menyuk, and P. K. A. Wai, "Application of the Manakov-PMD equation to studies of signal propagation in optical fibers with randomly varying birefringence," Journal of Lightwave Technology, vol. 15, no. 9, pp. 1735-1746, 1997.
[13] P. K. A. Wai and C. R. Menyak, "Polarization mode dispersion, decorrelation, and diffusion in optical fibers with randomly varying birefringence," Journal of Lightwave Technology, vol. 14, no. 2, pp. 148157, 1996.

[14] P. K. A. Wai, C. R. Menyuk, and H. H. Chen, "Stability of solitons in randomly varying birefringent fibers," Optics Letters, vol. 16, no. 16, pp. 1231-1233, 1991.

[15] S. Mumtaz, R. Essiambre, and G. P. Agrawal, "Nonlinear propagation in multimode and multicore fibers: Generalization of the Manakov equations," Journal of Lightwave Technology, vol. 31, no. 3, pp. 398406, 2013

[16] R. Ryf, S. Randel, A. H. Gnauck, C. Bolle, A. Sierra, S. Mumtaz, M. Esmaeelpour, E. C. Burrows, R. Essiambre, P. J. Winzer, D. W. Peckham, A. H. Mccurdy, and R. Lingle, "Mode-division multiplexing over $96 \mathrm{~km}$ of few-mode fiber using coherent 6x6 MIMO processing," Journal of Lightwave Technology, vol. 30, no. 4, pp. 521-531, 2012.

[17] D. Gloge, "Weakly guiding fibers," Applied Optics, vol. 10, no. 10, pp. 2252-2258, 1971.

[18] D. Gloge, "Dispersion in weakly guiding fibers," Applied Optics, vol. 10, no. 11 , pp. 2442-2445, 1971.

[19] M. J. Adams, An introduction to optical waveguides. UMI Books on Demand, 1981.

[20] A. Ghatak and K. Thyagarajan, Introduction to fiber optics. Cambridge university press, 1998.

[21] K. Iizuka, Elements of Photonics, vol. 2. John Wiley \& Sons, 2002.

[22] G. P. Agrawal, Nonlinear fiber optics. San Diego, CA: Academic, 4th ed., 2007.

[23] A. J. Lowery, L. B. Du, and J. Armstrong, "Performance of optical OFDM in ultralong-haul WDM lightwave systems," Journal of Lightwave Technology, vol. 25, no. 1, pp. 131-138, 2007.

[24] F. Ferreira, S. Jansen, P. Monteiro, and H. Silva, "Nonlinear semianalytical model for simulation of few-mode fiber transmission," Photonics Technology Letters, IEEE, vol. 24, no. 4, pp. 240-242, 2012.

[25] C. Koebele, M. Salsi, G. Charlet, and S. Bigo, "Nonlinear effects in mode-division-multiplexed transmission over few-mode optical fiber," Photonics Technology Letters, IEEE, vol. 23, no. 18, pp. 1316-1318, 2011.

[26] K.-P. Ho and J. M. Kahn, "Linear propagation effects in mode-division multiplexing systems," Journal of Lightwave Technology, vol. 32, no. 4, pp. 614-628, 2014. 